\title{
El Centro de Emprendedores de la Economía Social (CEES) de la ciudad de Santa Fe y su viabilidad de convertirse en un centro de referencia para la Economía Social de la región santafesina
}

The Center for Social Entrepreneurship and Social Economy in Santa Fe City and its Viability to Become a Model for Social Economy in the Region O Centro de Empreendedores da Economia Social (CEES) da cidade de Santa Fe e a sua viabilidade de se transformar em um centro de referência para a Economia Social da região santafesina.

Orlando Mariano Sotto

Facultad de Ciencias Jurídicas y Sociales, Universidad Nacional del Litoral, Argentina.

E-mail:sottoorlando@gmail.com

Fecha de recepción: 11/11/2016 Fecha de aceptación: 30/04/2017

\section{Resumen}

El presente trabajo tiene por objetivo analizar y profundizar el itinerario y las dinámicas desarrolladas en los últimos tres años en el Centro de Emprendedores de la Economía Social (CEES) de la ciudad de Santa Fe, la cual es una experiencia desplegada en forma conjunta por tres diferentes niveles del sector público: el Gobierno Local, el Provincial y la Universidad. Se apunta a indagar si las acciones y actividades desarrolladas por el conjunto de instituciones públicas colaboraron en la consolidación de las prácticas de Economía Social y Solidaria (ESyS) de la región.

La metodología utilizada ha sido principalmente técnicas cualitativas de investigación entre las que se incluyen: análisis y lectura bibliográfica, encuentros de trabajo y participación directa en los talleres formativos

Palabras clave

- Economía Social y Solidaria

- Políticas Públicas

- Emprendimientos socioproductivos

- Santa Fe realizados.

Entre los principales resultados se destacan: diseño, articulación, coordinación y evaluación de políticas públicas entre distintos organismos del Estado, fortalecimiento institucional y comercial de emprendedores 
(en mayor medida) y asociados de cooperativas de trabajo (en menor medida), mayor visibilidad institucional de las prácticas de ESyS y creación de un espacio de referencia para la ESyS en la región.

Con respecto a las conclusiones más relevantes, el CEES se está convirtiendo en una referencia para los emprendedores y cooperativas de trabajo locales, a través de acciones de capacitación, asesoramiento y seguimiento en diferentes temáticas, financiamiento y acceso a diferentes canales de comercialización. Con esos servicios, se busca apoyar la conformación de grupos asociativos y cooperativos con la intención de institucionalizar organizaciones de emprendedores; y generar un espacio propicio para el intercambio de experiencias. Asimismo se está gestando un espacio de gestión asociada entre el sector público y las experiencias de Economía Social de la ciudad, a partir de la planificación participativa.

\section{Abstract}

The aim of this work is to analyze and delve into the itinerary and the dynamics developed over the last three years in the Center for Social Entrepreneurship and Social Economy in Santa Fe City, which is an experience carried out jointly by three different levels of the public sector: the Local Government, the Provincial Government and the University. The objective is to investigate whether the actions and activities carried out by all public institutions contributed to the consolidation of Social and Solidarity Economy (SSE) practices in the region.

The methodology used has consisted mainly of qualitative research techniques including: analysis and reading of bibliography, working meetings and direct participation in the training workshops.

Among the main results are: design, interaction, coordination and evaluation of public policies among different government agencies; institutional and commercial strengthening of entrepreneurs (to a greater extent) and cooperative associates (to a lesser extent); greater institutional visibility of the SSE practices and creation of a model for SSE in the region, among others.

As regards the most relevant conclusions, the Center for Social Entrepreneurship and Social Economy is becoming a model for local entrepreneurs and labor cooperatives, through actions which include training, advice and monitoring on different topics, as well as financing and access to different commercialization channels. With these services, it seeks to support the formation of associative and cooperative groups with the intention of helping organizations of entrepreneurs to become institutio-

Keywords

- Social and Solidarity Economy

- Public Policies

- Social and Productive Enterprises

- Santa Fe nalized, as well as to create a favorable environment for the exchange of experiences. Moreover, through participatory planning, there is a new environment which enables joint management by the public sector and Social Economy experiences in the city. 


\begin{abstract}
Resumo
0 presente trabalho tem por objetivo analisar e aprofundar no itinerário e nas dinâmicas desenvolvidas nos últimos tres anos no Centro de Empreendedores da Economia Social (CEES) da cidade de Santa Fe, a que é uma experiência realizada em parceria por três diferentes níveis do setor público: 0 Governo municipal, o Provincial e a Universidade. 0 foco da pesquisa é indagar se as ações e atividades desenvolvidas pelo conjunto de instituições públicas colaboraram na consolidação das práticas de Economia Social e Solidária (ESyS) da região.

A metodologia utilizada tem sido principalmente técnicas qualitativas de pesquisa entre as que se incluem: análise e leitura bibliográfica, encontros de trabalho com referentes chave do CEES e com empreendedores e cooperativistas que participaram nas oficinas de capacitação e assessoria e de maneira direta nas formativas.

Entre os principais resultados destacam-se: desenho, articulação, coordenação e avaliação de políticas públicas entre diferentes organismos do Estado, fortalecimento institucional e comercial de empreendedores (em maior grau) e associados de cooperativas de trabalho (em menor grau), maior visibilidade institucional das práticas de ESyS e criação de um espaço de referência para a ESyS na região, entre outros.

Respeito das conclusões mais relevantes, 0 CEES está se transformando em uma referência para os empreendedores e cooperativas de trabalho locais, através de ações de capacitação, assessoria e seguimento em diversas temáticas, financiamento e acesso a diferentes canais de comercialização. Com esses serviços procura-se apoiar a conformação de grupos associativos e cooperativos com a intenção de institucionalizar

Palavras-chave - Economia Social e Solidária - Políticas Públicas - Empreendimentos sócio-produtivos

- Santa Fe organizações de empreendedores; e gerar um espaço apropriado para 0 intercâmbio de experiências. Também está se gestando um espaço de gestão associada entre 0 setor público e as experiências de Economia Social da cidade, a partir da planificação participativa.
\end{abstract}

\title{
1. Introducción
}

El presente trabajo tiene por objeto analizar y niveles del sector público: el Gobierno Local, el profundizar el itinerario y las dinámicas desa- Provincial y la Universidad. Se apunta a indagar rrolladas en los últimos 3 años en el Centro de si las acciones y actividades desarrolladas por Emprendedores de la Economía Social de la el conjunto de instituciones públicas colaboraron ciudad de Santa Fe, la cual es una experiencia en la consolidación de las prácticas de Economía desplegada en forma conjunta por tres diferentes Social (ES) de la región. 


\subsection{Importancia del tema}

La ciudad de Santa Fe y su zona de influencia tienen indicadores sociales de pobreza, desempleo e indigencia elevados con respecto a otros lugares de la Argentina, ${ }^{1}$ que se corresponden con lo que acontece a nivel del país. Esto marca el grado de vulnerabilidad de gran parte de la sociedad local y el escaso nivel de integración de la misma dadas las grandes dificultades existentes en la actualidad, tanto de tipo económico (desempleo, exclusión, trabajo informal, cuentapropismo,etc.), como de tipo social (altos niveles de delincuencia, indigencia, bajos niveles educativos en gran parte de la población, violencia familiar, de género, analfabetismo, exclusión social, inseguridad, etcétera).

Desde el enfoque de la Economía Social y Solidaria (en adelante ESyS), en la ciudad de Santa Fe y su zona de influencia, las experiencias que han sido puestas en marcha por parte del Estado local, provincial y el universitario ${ }^{2}$ se han incrementado en los últimos años. Por ejemplo, la creación en 2015 de una Dirección de Economía Social en la Municipalidad de Santa Fe, de una Secretaría a nivel provincial (con sede en la ciudad de Santa $\mathrm{Fe}$ ), y no sólo la profundización en el trabajo de campo sino también la aprobación y ejecución de un Programa de Extensión de Economía Social y Solidaria por parte de la UNL, buscan impulsar, estimular, fortalecer y difundir el movimiento de la ESyS en la ciudad.

El Centro de Emprendedores de la Economía Social (CEES) es un espacio de articulación política cogestionado entre la Municipalidad de Santa Fe, el Gobierno de Santa Fe y la Universidad Nacional del Litoral y se constituye como ámbito de referencia para promover, cooperar y colaborar con agentes, instituciones y organismos públicos y privados en el diseño y ejecución de procesos y estrategias de fortalecimiento de la Economía Social en la ciudad de Santa Fe.

Sus principales objetivos son:

- Brindar capacitación y asistencia técnica.

- Constituir un espacio de encuentro e intercambio de experiencias entre emprendedores.

- Promover la comercialización en el mercado local y regional.

- Vincular líneas de financiamiento para emprendedores y proyectos de la Economía Social.

- Diseñar mecanismos de evaluación que permitan medir impactos, resultados logrados, obstáculos, apropiación de tecnologías y conocimientos en el marco del proceso productivo.

- Propiciar el fortalecimiento de los emprendedores/as como grupo de acción colectiva, fomentando instancias de diálogo y debate que contribuyan a dar representatividad a sus intereses en el ámbito del Centro de Emprendedores (UNL, s.f.).

Por la tanto, con el surgimiento del CEES las experiencias e instituciones de Economía Social de la ciudad y la región no solo tienen la posibilidad de tener un lugar de encuentro y de reconocimiento, sino también una plataforma a partir de la cual institucionalizar, reconocer, capacitar y capitalizar el movimiento de ESyS que se encuentra en esta parte de la provincia de Santa Fe (Argentina).

\section{Objetivos}

Este artículo pretende describir y estudiar el trayecto desarrollado desde el año 2013 a la actualidad por el CEES, el cual busca ser una plataforma

(1) Según el INDEC (Instituto Nacional de Estadísticas y Censos), para el I trimestre de 2016, el Gran Santa Fe tenía una tasa de desocupación del $14 \%$ y en el ler semestre el nivel de pobreza alcanza el 18.4 $\%$ y la indigencia el 6.5\%. (INDEC, 2016)

(2) De estas experiencias que se han implementado en los últimos años gran parte de ellas no se encontraban relacionadas, aspecto que en los últimos años se viene modificando ya que han surgido experiencias colectivas de ESyS (el CEES, el Camión de la Economía Popular, ferias autogestionadas, proyectos de extensión desarrollados desde las universidades, entre otros) 
de referencia, fortalecimiento y seguimiento de las prácticas de la Economía Social, mediante la generación de proyectos y experiencias concretas para un modelo de desarrollo sustentable, inclusivo, integral, participativo, sostenible y con equidad social. Entre los principales objetivos se incluyen:

- Describir y analizar algunas de las acciones y actividades implementadas desde el CEES y su influencia en la consolidación de las prácticas de Economía Social y Solidaria de la ciudad de Santa Fe.

- Indagar si las medidas desarrolladas por cada uno de los niveles del Estado involucrados en el CEES puede ser inferidas como políticas de Economía Social.

En cuanto a la hipótesis de trabajo la misma se define de la siguiente manera «no es posible el desarrollo y fortalecimiento de la Economía Social y Solidaria sin un Estado en sus distintos niveles (local, provincial y nacional) y de instituciones del conocimiento técnico y científico que acompañen y asisten adecuadamente y en forma permanente al movimiento de la ESyS».

\section{Marco teórico}

\section{a) Descripción de la evolución} del sistema capitalista mundial

El mundo está atravesando actualmente por una crisis civilizatoria inédita, donde se pone en riesgo para las próximas décadas la propia supervivencia del ser humano en el planeta. El agotamiento del modelo fordista-keynesiano (1949/50-1970/75) que permitió al sistema capitalista internacional un largo período de crecimiento durante la segunda posguerra, desató fuertes reacciones desde mediados de los años 70, promovidas por los sectores más concentrados de los países centrales, que apuntaron a una «reconversión» del propio sistema para recuperar la caída en la tasa de ganancia y las posibilidades de una nueva fase expansiva de largo plazo.

En un contexto finito como lo es nuestro planeta, las tendencias históricas del sistema comenzaron a poner en evidencia hacia finales del siglo XX los límites severos que enfrenta este modo de organización social para resolver en su marco los grandes problemas de la humanidad. La necesidad de ampliar en forma permanente los mercados choca con una población mundial mayoritariamente empobrecida, y con la necesidad de los actores más poderosos (las grandes empresas transnacionales) de crecer a costa de «depredar» los mercados ya existentes.

Para ello, el neoliberalismo como proyecto político y como política de Estado promovió el apoderamiento masivo de sectores económicos (y mercados) que estaban ocupados por otros actores.

Simultáneamente al avance y control de los mercados por las megas empresas a escala mundial, se observa otro proceso altamente preocupante desde el punto de vista social: el desempleo. Con la organización en gran escala a partir de las nuevas tecnologías, esas actividades son ahorradoras y expulsoras de inmensas cantidades de mano de obra. Eso significa que también se reducen drásticamente las posibilidades de ingresos necesarios para la vida, para millones y millones de personas que van siendo arrojadas al desempleo y a la marginalidad. Esto sucede en las más variadas actividades económicas (industria, energía, sector primario, comercio, servicios, etcétera).

\section{b) La economía social como camino alternativo a desandar y construir}

Es necesario pensar en forma estratégica en el impulso de un cambio estructural del contexto, de manera de ir conformando un espacio sistémico que genere en forma progresiva una independencia de las lógicas impuestas por la competencia y por las leyes del mercado salvaje.

En este sentido, las tendencias del sistema antes mencionadas nos muestran que no existen posibilidades de un desarrollo sustentable de 
emprendimientos individuales o colectivos de la Economía Social y Solidaria en el marco adverso del sistema capitalista (en especial en su actual etapa neoliberal).

Se requiere para ello pensar en el desarrollo de un sector de la economía social y solidaria que esté liberado de la lógica capitalista de la competencia y la máxima ganancia, y de la eficiencia y la eficacia medidas en función de aquéllas; orientar, en cambio, los recursos y las capacidades disponibles para resolver las necesidades de las personas (la reproducción de sus miembros y comunidades de referencia), y a la vez generar nuevos valores alternativos al egoísmo individual, la competencia desenfrenada y la acumulación de riquezas ilimitada. Esto implica un proceso prolongado de cambio social, donde deben ir cambiando las instituciones, la cultura, las relaciones de poder, y surgiendo nuevos sujetos colectivos comprometidos con esa transformación (Coraggio, 2010:52).

Para avanzar en ese camino las familias e instituciones comprometidas (asociaciones, universidades, etcétera) con esta transformación deberían buscar producir bienes y servicios que cubran sus propias necesidades (producción para el autoconsumo 0 para el intercambio intra sector); procurar lograr mayores ingresos en su intercambio 'externo' con el sector capitalista y con las pymes (mejorando en este caso su capacidad competitiva sin afectar sus principios y valores); a la vez que desarrollando relaciones comunitarias y sociales que fortalezcan un estilo de vida que se oriente hacia una nueva sociedad (Tealdo, 2013:4).

Citando a Coraggio (2004:22):

Hoy, el problema más acuciante para decenas de millones de latinoamericanos es comer y vestirse, pero también vuelve a ser necesidad insatisfecha refugiarse, transportarse, acceder a la salud, obtener tarifas sociales de servicios indispensables, acceder a la educación secundaria y continua, y recuperar espacios de sociabilidad y contención afectiva. Este conjunto de necesidades, el verdadero objetivo de la economía, reclama la organización solidaria de las unidades domésticas de los trabajadores y trabajadoras para que organicen sistemas de seguridad alimentaria, compren juntos para aumentar su poder en los mercados, intercambien trabajos fuera del mercado monetizado como en las redes de trueque (...) Reclama también que el sistema científico tecnológico, las universidades e institutos terciarios y el sistema educativo público en general, se conecten con las necesidades de aprendizaje de los agentes y organizaciones de este sector de la economía.

\section{c) Conceptualizando a la Economía Social y Solidaria (ESyS)}

José Luis Coraggio define a la ESyS como «un sistema de organización de la producción y división social del trabajo, de circulación y distribución y de legitimación de las formas de satisfacción de necesidades, centrado en el trabajo libremente asociado, con un sujeto sociopolítico articulador de los distintos movimientos que pugnan por la reproducción ampliada de la vida de las personas y comunidades, opuesto al capital» (Hintze, 2007:15). Si se asume esta concepción como punto de partida, una categorización de las prácticas de Economía Social debería contener a iniciativas que: a) Estén centradas en el trabajo; b) Se realicen libremente asociadas; c) Persigan como fin la reproducción (ampliada) de la vida de los miembros; d) Donde la libre asociación se lleve a cabo para la resolución de necesidades comunes.

Producto de la crisis de reproducción resultante de un largo proceso histórico de empobrecimiento estructural en el contexto de políticas económicas y sociales neoliberales, los sectores populares, sus unidades domésticas y organizaciones, vienen desarrollando iniciativas económicas, cuyo sentido es la obtención de medios de vida para resolver las necesidades más acuciantes, los cuales constituyen un sustrato inorgánico del sistema económico, que el mismo autor denomina de Economía Popular, absorbido por una dualidad entre comportamientos competitivos y particularistas y lógicas de coopera- 
ción y reciprocidad con diversos alcances (cooperativas, mutuales, asociaciones, etcétera).

Pensar en la Economía Social y Solidaria implica por tanto, establecer un horizonte de transformación del actual sistema económico.

Se puede expresar que hay autores con un enfoque donde la Economía Social es entendida que surge por un problema coyuntural, de una crisis socioeconómica profunda (Nosetto, 2013:3; Arroyo, 2013:7). Este enfoque, llamado de Economía Social, considera a la misma como la economía que surge cuando el sistema de mercado capitalista excluye a un sector de la población al que, a su vez, el Estado tampoco da respuestas; entonces aquellos que se encuentran en ese sector comienzan a resolver sus necesidades básicas por mecanismos propios de solidaridad, trueque, autoconsumo, intercambios, etc. Aquí el mercado es lo natural (Nosetto, 2013:3).

Otra concepción hace referencia a aquella partícipe de otro tipo de economía, diferente de la del mercado y la economía del Estado, como un proyecto diferente del neoliberal, un proyecto nacional de desarrollo distinto, un modelo inclusivo (García Delgado,2013:3). Tiene que ver con la posibilidad de que estas unidades económicas se vinculen con un proyecto que junto al Mercado y al Estado puedan constituir un sistema plural donde el trabajo esté sobre el capital, donde la reproducción ampliada de la vida sea priorizada a la reproducción del capital (Coraggio, 2007:54).

La tercera acepción, es la que se refiere a la Economía Social, como complemento o partícipe de otro tipo de economía, como es la del mercado y la economía del Estado. Este enfoque se enmarcaría en la teoría de Karl Polanyi (1989). El planteo es un sistema económico plural donde conviven estos tres subsistemas (el privado, el público y el social) y donde lo social tenga una dimensión preeminente.

Aquí la economía social actúa como un subsistema (subsistema social), que convive con la Economía de Mercado (subsistema privado), y con la economía del Estado (subsistema público). Estaríamos hablando de un sistema económico plural,
Mercado, Estado y lo social, lo que otros autores llaman el tercer sector, Sociedad Civil, Estado y Mercado. Hablarde la economía social en este enfoque, es considerarla como complemento de las actividades lucrativas (economía de mercado), donde hay sectores o nichos marginales, donde no es posible desarrollar una economía de mercado o estatal, ya sea por la ubicación geográfica, territorial, por el sector económico, por el tipo de personas, etc., y es allí que surge la economía social como complemento. En este enfoque el sistema económico estaría conformado por la economía empresarial capitalista de mercado, la economía pública estatal y la economía popular. Donde cada una de ellas funcionarían con su particularidad, la economía de mercado con la máxima ganancia, la economía pública acumular poder estatal para la gobernabilidad, y la economía popular cuya lógica fundamental es la reproducción de la vida, mejorar la calidad de vida. Aquí las organizaciones podrán funcionar llenando los espacios dejados por el capitalismo por no ser rentables (empresas recuperadas), en forma autónoma, aislada y desconectada del mercado formal y una más radical que buscaría la superación de la misma, en base a la confrontación no solo desde lo teórico ideológico sino desde las propias prácticas productivas. Se plantea como una variante de «economía plural» como etapa de tránsito, donde previamente hay que saldar discusiones en torno al papel del Estado, la organización del mercado, el dinero y sus funciones, el régimen de propiedad y los valores que den sustento a otra economía y la plantea la necesidad de construir un sector orgánico de la economía social «en base al desarrollo, agregación y articulación de organizaciones económicas con relaciones de producción no capitalistas, que coexistiría y/o pugnaría, en una economía mixta, con las lógicas y organizaciones del capital y del estado»(Coraggio, 2005:3).

Para el presente artículo se toma el concepto de que toda economía es social, ya que las actividades económicas que implican el proceso de producción y distribución son realizadas a partir de las relaciones 
que establecen los hombres, se lleva a cabo en base un tipo de vínculo socioeconómico y, en este marco,debe ser solidaria, porque entraña un tipo de relación no competitiva, no individual, como se plantea en la economía capitalista.

Se entiende, entonces, que la ESyS es un proceso continuo en construcción y fortalecimiento, que actualmente se desarrolla o coexiste con el Estado y el mercado.

Es por esto que el concepto elegido para el presente texto se vincula con la última concepción enunciada, en la cual existen y conviven tres subsistemas diferentes y, dependiendo de las particularidades históricas, uno tendrá prevalencia sobre los otros. Desde este enfoque se considera que la ESyS es el próximo subsistema que tendrá prevalencia sobre los otros dos, en la historia por venir.

\section{d) Metodología y técnicas de investigación}

La metodología aplicada en el presente artículo ha sido la siguiente:

- Recolección, lectura, análisis y comparación del material bibliográfico.

- Entrevistas semiestructuradas con los actores relevantes e informantes clave, entre los que se incluyen los coordinadores de los diferentes organismos públicos involucrados y emprendedores sociales.

- Participación en los talleres y capacitaciones realizadas en el CEES.

- Observación participante en el dictado de capacitaciones que se realizaron en el CEES.

\section{e) Breve historia del Centro de Emprendedores de la Economía Social (CEES)}

El CEES es un espacio cogestionado entre la Universidad Nacional del Litoral, la Secretaría de Desarrollo Territorial y Economía Social de la Provincia de Santa Fe y la Dirección de Economía Social del Municipio local, constituido con la finalidad de articular, promover y cooperar con agentes, instituciones y prácticas en el diseño y ejecución de procesos y estrategias de Economía Social de la ciudad de Santa Fe.

Producto de la articulación institucional, se han vinculado a su labor cotidiana alrededor de 1000 emprendimientos inscriptos en el Registro de Emprendedores Locales del Municipio, en su mayoría individuales o familiares, con características, comportamientos y trayectorias heterogéneas.

Las entidades públicas mencionadas inauguraron el CEES el 14 de mayo de 2013. En dicha oportunidad encabezaron el acto la ministra de Desarrollo Social, Mónica Bifarello, el intendente de la ciudad, José Corral, y el vicerrector de la Universidad Nacional del Litoral, Miguel Yrigoyen. En la oportunidad, la Ministra expresó: «Estamos inaugurando el Centro de Emprendedores de la ciudad de Santa Fe que seguramente también va a tener influencia sobre toda la región. Es un proyecto que hace mucho tiempo venimos trabajando desde el Gobierno de la provincia con la Municipalidad de Santa Fe y con la Universidad. (...) La idea es que los emprendedores de la ciudad y la región, tengan su espacio físico, este espacio va a estar destinado a encuentros, intercambios, capacitaciones» (Gobierno de Santa Fe, 2013).

Se concibe desde este espacio una mirada plural de la economía social, donde la política pública debe superar la simple asistencia en la promoción de microemprendimientos de los sectores más vulnerables. Se piensa a la economía social, como un horizonte al cual arribar a través del fortalecimiento de distintas prácticas productivas, que les permita adquirir la sostenibilidad suficiente para desarrollarse en otros valores, entre ellos el asociativismo.

La iniciativa comprende el otorgamiento de microcréditos a través de un Banco Solidario. En el CEES, los emprendedores acceden a capacitación, asesoramiento, financiamiento y a diferentes canales de comercialización.En ese sentido, la asistencia técnica y las capacitaciones están orientadas en primera instancia a cuatro áreas: Formación General 
(Asociativismo, Planificación, Gestión, Contabilidad, Precios, Comunicación, Costos, Ventas, estrategias de comercialización, Plan de Acción Sustentable); Formaciones específicas en temáticas relacionadas con la producción; Servicios generales (inscripciones y formalización, entre otros); y acompañamiento en el proceso asociativo y de formación de espacios de representatividad colectiva.

El público al que están dirigidas la mayor parte de las acciones del CEES está conformado por pequeños emprendimientos productivos, en su mayoría unipersonales o familiares, formales o informales (en la generalidad de los casos de esta última forma), que en gran parte surgen como estrategias de los hogares de trabajadores excluidos del empleo asalariado como necesidad de dar respuesta a las demandas reproductivas del grupo al que pertenecen (familiar en su mayoría), y en donde el trabajo se constituye como su recurso principal; presentan además un escaso acceso a tecnologías y dificultades de financiamiento. Estas experiencias forman parte de los sectores populares, de distintos barrios de la ciudad, con importantes necesidades de fortalecimiento de su actividad productiva (Mejías, 2014:2).

En términos socioeconómicos, estas unidades presentan características heterogéneas: algunas están más vinculadas a la subsistencia, otras con algunas dificultades más concretas (acceso al financiamiento, limitaciones funcionales $y / 0$ estéticas de los productos o servicios ofrecidos, entre otros), y un tercer grupo minoritario conformado por aquellos que poseen estas unidades como actividad complementaria a su actividad principal - y sin necesidades económicas de sostenerlas-, desarrolladas por razones de índole personal (Massera, 2015:7).

\section{Análisis, resultados y discusión del CEES}

Teniendo en cuenta el concepto de Economía Social expresado para el presente trabajo en el marco teórico, se describirán un conjunto de actividades y acciones llevadas adelante por el gobierno de la Ciudad de Santa Fe, el gobierno de la Provincia homónimay la Universidad Nacional del Litoral (UNL) que se siguen llevando a cabo con el objeto deafianzar las prácticas y expresiones de la Economía Social y a su vez contribuir a construir otro tipo de Economía.

Más allá de estas diferenciaciones, pueden reconocerse algunas características comunes en todas ellas:

- El trabajo se constituye en todos los casos como el factor o recurso principal en su actividad.

- La remuneración del trabajo es directamente proporcional a la cantidad de trabajo incorporado en ellas.

- Se observa una inestabilidad en la calidad del trabajo, con escasa o nula cobertura de seguridad social y bajos niveles de ingresos.

- Presentan dificultades de acceso a financiamiento, ya sea por excesivos requerimientos de las instituciones financieras o por no adecuarse a las necesidades de este sector.

- Presentan dificultades para la comercialización, ya sea en el suministro de insumos de producción (acceden a insumos productivos más caros que las empresas), como en la venta (sólo cuentan con espacios de ferias y ventas al menudeo, no se originan demandas al sector, y existe cierta reticencia del público en relación con la calidad de sus productos).

- Presentan problemas de asistencia técnica y capacitación, dependiendo en general de los espacios que promueven las instituciones públicas para el abordaje de estos aspectos (Municipalidad, Provincia, o el propio CEES) (Mejías, 2014:8).

Cada una de estas cuestiones representan desafíos por ser superados, es por ello que desde el CEES se desarrollaron y se continúan realizando una serie de iniciativas tendientes a resolver algunos de los mismos, teniendo en ciertos casos una visión más cortoplacista y en otras una visión de construir 
una mirada colectiva de largo plazo (aspecto fundamental para construir prácticas de ESyS sostenibles y que permitan consolidar dicho subsistema económico). En lo siguiente se detallan algunas de las actividades desarrolladas en el CEES.

\section{a) Actividades desarrolladas en el CEES}

Estas unidades encuentran como primer contacto con el CEES - y obligatorio para acceder a cualquier otro servicio - el Registro de Emprendedores Productores Locales, el cual es un registro que lleva adelante la Municipalidad de la ciudad de Santa Fe en el espacio del Centro de Emprendedores, y en el cual pueden inscribirse todas aquellas personas que sean titulares de un emprendimiento individual 0 asociativo, siempre y cuando sea de carácter productivo. Éste registro existía con anterioridad al surgimiento de este espacio, pero la cantidad de emprendimientos registrados creció notablemente desde ese momento. Desde su inauguración, en mayo del 2013, se han entregado 450 credenciales de emprendedores, siendo al día de hoy 780 los emprendedores en la ciudad de Santa Fe que se encuentran en actividad, cuyos rubros principales son la carpintería, alimenticio, textil, herrería, artesanías y jardinería (Massera,2015:8).

A partir de esta inscripción, los emprendedores pueden optar por insertarse en algunos de los tres ejes principales que desarrolla el CEES: capacitaciones, financiamiento y espacios de comercialización.

Con respecto a las capacitaciones proporcionadas se dividen en 3 grandes grupos:

- Curso de Formación Integral: una de las prioridades tenidas en cuenta al comienzo del funcionamiento del CEES fue atender las demandas específicas de los emprendedores sociales con respecto a capacitaciones en Costos, Comercialización, Planificación Estratégica e Identidad Visual. De esta forma, se desarrollaron y desarrollan talleres de Formación
General en estas temáticas a cargo de docentes especializados y que son parte del equipo de Economía Social y Solidaria de la UNL. Cabe destacar que en las capacitaciones efectuadas se incluyen conceptos como Precio Justo, Consumo Responsable, Compras y Ventas Conjuntas, de manera que los emprendedores vayan tomando conocimiento sobre otras formas alternativas de hacer intercambios entre personas, las cuales son importantes para generar las bases para Otra Economía.

- Curso de Formación Inicial: destinado a emprendedores que recién han comenzado con su emprendimiento social o que tienen una idea de una iniciativa en mente. Tiene menos encuentros de trabajo que el Integral y se realiza una introducción muy general en las temáticas de Administración, Comercialización y Costos, a partir de las cuales los emprendedores puedan empezar a organizar su iniciativa. Para poder profundizar estos tópicos los emprendedores deben inscribirse para realizar el Curso Integral (explicado anteriormente), en el cual se proporcionan más herramientas organizacionales y su aplicabilidad en las iniciativas productivas.

- Cursos satélites: surgen debido a la demandas de talleres específicos que realizan los distintos emprendimientos. Hay que tener en cuenta que la heterogeneidad de las prácticas de ESyS, Ileva a que las demandas de capacitación también contengan estas características. Es por esto que desde el CEES se dictan talleres cortos de fotografía, serigrafía, uso de redes sociales, entre otros. Asimismo, debido a la diversidad en las características de los emprendimientos, la oferta de cursos satélites o específicos varía de semestre en semestre.

Es importante señalar que estos talleres de formación implementados desde el CEES han tenido un impacto positivo en las distintas iniciativas productivas, según las valoraciones realizadas por los propios emprendedores. Entre las mismas se pueden mencionar: que sus demandas fueron atendidas, que tienen un espacio de referencia para trabajar en forma colectiva y a partir del cual 
fortalecer su emprendimiento, que han tomado conciencia de lo importante que es organizar la comercialización y los costos del emprendimiento, la posibilidad de aprender cosas nuevas. De esta manera se ha contribuido al fortalecimiento organizacional y comercial de muchos emprendimientos.

Estos cursos no solo tienen como finalidad brindar conocimientos técnicos sino también empezar a generar transformaciones culturales en los emprendedores, ya que se apunta a que tengan un enfoque más horizontal y solidario de su actividad productiva. Este es un proceso de mediano plazo que deber ser acompañado y respaldado con otras acciones desde el CEES, siempre y cuando se cuente con la predisposición de los emprendedores.

Estos encuentros de formación que se continúan haciendo, constituyen una plataforma de trabajo muy importante para el afianzamiento de la ESyS de la ciudad de Santa Fe, puesto que se trabaja atendiendo demandas e inquietudes expresadas por los emprendedores, tratando de atender a la mismas de forma dinámica, asociativa y flexible entre los organismos públicos involucrados en el CEES, aspecto fundamental para que no solo las expresiones de la Economía Social se fortalezcan en la ciudad de Santa Fe, sino también el trabajo articulado e integrado entre los actores de la sociedad civil y el Estado. Asimismo,esta actividad ha tenido como resultado más concreto la reorganización interna de cada emprendimiento, trabajando en una escala más microeconómica e individual.

Si se pretende afianzar a las expresiones de la ESyS en la región es necesario y fundamental pensar, diseñar, implementar y trabajar en la construcción de procesos colectivos de mediano y largo plazo, tanto en los actores de la sociedad civil como en los organismos públicos participantes en el CEES. Este es un aspecto que los responsables del CEES deberían tener en cuenta para trabajar no sólo en la dimensión micro de la ESyS, sino también, al decir de Coraggio, en el nivel mesoeconómico (cabe mencionar que en los últimos meses esta cuestión venía siendo planteada desde el sector universitario).
Otra de las iniciativas desarrolladas desde el CEES están vinculadas con cooperativas de trabajo de la ciudad de Santa Fe, las cuales forman parte del conjunto de prácticas de la ESyS de la ciudad de Santa Fe.

\section{Capacitaciones con Cooperativas de Trabajo}

En este caso puntual se ha desarrollado en el año 2015, un taller denominado «Asociativismo y Autogestión», el cual está destinado a asociados de cooperativas de trabajo y que tiene por finalidad introducir nociones básicas sobre el funcionamiento y organización de una cooperativa y trabajar en torno al concepto de Economía Social dentro de la misma. Del mismo participaron asociados de distintas cooperativas de trabajo, los cuales tuvieron la oportunidad de aprender sobre la tarea y labor administrativa y legal de la cooperativa y de algunas nociones básicas de Economía Social.

Por otro lado en el año 2016 se ha desarrollado el Curso de «Fortalecimiento de Cooperativas de Trabajo recientemente constituidas», realizado por el Gobierno de Santa Fe, con la colaboración del Programa de Extensión de Economía Social de la UNL, el cual tiene como objetivo abordar las problemáticas inherentes al funcionamiento de cooperativas de trabajo, tanto sea en cuestiones referidas a la actividad formal de acuerdo con la normativa vigente, como a las relaciones interpersonales entre los asociados y sus vínculos asociativos y que está destinado a asociados de cooperativas de trabajo constituidas recientemente.

Ambos espacios de trabajo han sido muy bien recibidos por los asociados de las cooperativas, puesto que les han permitido aprender cuestiones relativas a la organización y administración de la misma, las responsabilidades de cada uno mismos dependiendo el cargo que ocupe, la importancia de darse un momento para conversar sobre lo que significa Economía Social y también para conocer a otras experiencias cooperativas. Asimismo los asociados de las cooperativas, continúan con distintas demandas hacia el CEES, como ser espa- 
cios de asesoramiento comercial, impositivo y legal, así como alguna posibilidad de obtener financiamientos 0 subsidios para poder adquirir maquinas, equipos y para capital de trabajo.

En cuanto a la contribución de estas acciones al movimiento de la Economía Social de la ciudad se puede considerar positivo ya que muchas de las cooperativas se han podido conocer y han realizado algunos trabajos en forma colectiva. También ha permitido que se organicen parte de las cooperativas que participaron de los talleres para formar una Federación de Cooperativas (la cual está por obtener su matrícula nacional para poder empezar a funcionar), por lo cual la dimensión asociativa se ha visto fortalecida.

\section{b) EI CEES como impulsor de la Economía Social de la ciudad de Santa Fe}

Si bien los representantes de los organismos públicos involucrados trabajan para lograr la finalidad con la cual fue creado el CEES, como un ámbito en el que se generen y fortalezcan practicas asociativas, estos coinciden en que dichas experiencias colectivas no han avanzado mucho entre los emprendedores - aunque valoran los intentos que se han realizado en esa dirección-. Cabe destacar que si bien hay cooperativas vinculadas a actividades realizadas desde el CEES, una de las líneas institucionales de intervención del mismo, es la formación de grupos asociativos de emprendedores o personas que deseen desarrollar su actividad en común.

Como se ha expresado en el artículo, muchas de las acciones desarrolladas han apuntado al afianzamiento individual de cada emprendedor, buscando fortalecer su capacidad organizativa y comercial,y no han consolidado su capacidad asociativa. Este proceso desarrollado que ha tendido al individualismo, dificulta la consolidación de la ESyS en la ciudad de Santa Fe, puesto que para que el movimiento se pueda afianzar es fundamental no solo la presencia de los actores públicos que vienen pensando, impulsando y sosteniendo esta experiencia inédita en la provincia, sino también pensar, diseñar e implementar estrategias colectivas entre los cooperativistas y emprendedores involucrados en el CEES. Por eso también son necesarias acciones que promuevan el acercamiento de las cooperativas de la ciudad de Santa Fe, como ser Mesas o Encuentros de Trabajo, Rondas de Negocios, en las cuales los organismos públicos pueden poner en contacto empresas del sector privado con cooperativas de trabajo.

Uno de los coordinadores del CEES piensa que es necesario e imprescindible continuar y consolidar la dimensión asociativa, tanto de los emprendedores como de los cooperativistas, para la cual hay que generar ámbitos de trabajo e intercambio en los cuales la asociatividad se pueda construir y pensar. También sostiene que mucha de las acciones ideadas y realizadas en el CEES — como los espacios de capacitación, de comercialización, otorgamiento de subsidios, entre otras - dificultan y vuelven más lentos estos procesos. En esta situación una alternativa para poder avanzar en el proceso de asociatividad es reflexionar las actividades del CEES más allá de las urgencias políticas, institucionales y económicas. Cabe destacar que en esta alternativa la Universidad Nacional del Litoralpuede y debe tener un papel relevante y coordinador, ya que cuenta con la capacidad para articular y vincular a todas las partes involucradas, contribuyendo de esta manera a que el CEES se puede consolidar como la plataforma de la Economía Social de la ciudad de Santa Fe.

Otro de los que fue coordinadores responsables del CEES, destaco como un resultado muy importante el hecho de que los emprendedores y cooperativistas de la ciudad tienen un lugar de referencia, encuentro, capacitación, formación y debate de la ESyS. Asimismo subrayo que desde la implementación del CEES estas experiencias y expresiones de la ESyS tienen no solo una mayor visibilización en la comunidad santafesina, sino también un lugar 
de consulta, asesoramiento y apoyo continuo, lo y para mostrarles a los emprendedores y coopeque permite atender, en la medida de las posibili- rativistas que el trabajo asociativo y articulado es dades, las inquietudes y consultas que van reali- posible. Como cita un viejo refrán, «el mejor ejemplo zando y llevando al CEES los actores de la Economía empieza por casa». Por esto el trabajo colectivo y Social. Más allá de comprender a los emprendi- coordinado de los actores públicos involucrados en mientos asociativos como aquellos que mayores el CEES es imprescindible, ya que los organismos potencialidades y oportunidades presentan para públicos se deben poner como ejemplo de trabajo transformarse en sujetos activos de la construc- asociado y marcar el rumbo que debe seguir la ESyS ción de una economía social, el haber recuperado de la ciudad.

habilidades y capacidades productivas de grupos de personas individualmente, que de otra forma se verían excluidas de ponerlas en funcionamiento, ya es considerado como un objetivo cumplido del CEES (Massera, 2015:12).

Estas dos declaraciones son importantes, ya que permiten realizar una comparación de las ideas y lógicas de los coordinadores responsables del CEES. Como se puede apreciar, uno de los responsables tiene una lógica más individualista y de corto plazo, lo que genera ciertas dificultades a la hora de pensar la construcción de un movimiento más horizontal, colectivo, inclusivo e integrador de las experiencias de ESyS de la ciudad.Este aspecto contribuye a comprender la razón por la cual muchas de las actividades y acciones efectuadas desde el CEES tienen por objetivo organizar y afianzar a emprendedores individuales 0 alguna cooperativa en particular, brindándoles herramientas necesarias para que salgan a competir de la mejor manera posible en cada uno de los mercados de sus productos. Por otro lado, la visión del otro coordinador del CEES apunta a una mirada más horizontal y colectiva, tratando de generar los espacios para poder trabajar y concientizar tanto a los emprendedores como a los cooperativistas sobre la relevancia y necesidad de la dimensión asociativa y solidaria de la ESyS. Si se pretende que el CEES se convierta en un espacio de referencia, trabajo, seguimiento, encuentro y formación de la ESyS en la ciudad de Santa Fe es fundamental conciliar las puntos de vista y las lógicas de trabajo de los representantes de los organismos públicos involucrados en el CEES, para coordinar y aunar esfuerzos en la misma dirección,
Asimismo, se reflexiona que si bien se ha incrementado la visibilización de estas iniciativas productivas en la ciudad de Santa Fe, y las mismas han logrado un mayor reconocimiento de la comunidad santafesina, sigue siendo prioritaria la necesidad de planificar y reconstruir su identidad, su finalidad, sus objetivos, sus formas organizativas y su reconocimiento legal. El CEES puede convertirse en una plataforma desde la cual configurar esta identidad, ya que con el tiempo ha habido emprendedores y cooperativistas que se han apropiado e identificado con este espacio (algunos en mayor medida que otros), lo sienten como un lugar de encuentro, debate, discusión e identificación de pares, intentando la cimentación de una identidad colectiva entre los mismos, primordial para la consolidación de la Economía Social y Solidaria en la ciudad. Los emprendedores y cooperativistas que son parte de este contingente identificado con el CEES han participado en todas las acciones que desde el CEES se han venido implementando (talleres de formación, espacios de comercialización, Banco Solidario), lo que es una variable a tener en cuenta para la elaboración de actividades que apunten a fortalecer el movimiento de la ESyS en Santa Fe. El hecho de que hayan compartido diferentes espacios de capacitación y encuentro ha permitido que se comiencen a reconocer como pares y que visualicen a la organización colectiva como una manera de fortalecerse mutuamente.

Por otro lado, con todas las acciones que se han venido ejecutando desde el CEES en los últimos 3 años, la valoración, la capacidad de organización, de decisión y de establecer vínculos de confianza, 
pilares fundamentales para el trabajo participativo y asociativo, se han venido desarrollando y fortaleciendo. Estos aspectos son sumamente importantes para el afianzamiento de la ESyS en la región.

Cabe aclarar que al CEES en el presente artículo se considera dentro de la Economía Social porque es una iniciativa organizada por tres niveles del Estado y coordinada con actores de la sociedad civil, en este caso los emprendedores sociales (en mayor medida) y asociados de cooperativas (en menor medida), la cual busca estar basada en el esfuerzo propio, en la ayuda y confianza mutua, en la solidaridad, en la reciprocidad, en la igualdad de derechos y deberes, en principios construidos colectivamente y reafirmados constantemente mediante encuentros periódicos entre todos los actores e instituciones participantes.

Hay que destacar que el espacio colectivoque se está cimentando en el CEES no persigue la finalidad del lucro, sino que apunta a colaborar y a generar las condiciones para que los emprendedores y cooperativistas se generen los recursos necesarios para recuperar el capital de trabajo invertido en el emprendimiento y para asegurar la reproducción ampliada de sus familias; busca fortalecer la autogestión y al fomento de un comercio responsable, basado en la difusión de los fines constitutivos, el origen del producto, la dimensión territorial y la generación delexcedente para la propia comunidad. Todos estos elementos contribuyen a reflexionar que el crecimiento, desarrollo y consolidación de la ESyS es posible en el largo plazo, ya que se visualizan valores, principios y prácticas, que son constitutivos de esta otra economía, al decir de Coraggio. En este espacio multiactoral (a pesar de sus limitaciones, sus contradicciones, los aspectos que falta resolver, los desafíos por superar, las acciones por coordinar) los actores involucrados se consideran como integrantes de la ESyS y tratan de llevar a la práctica, con toda la complejidad y los desafíos que eso implica, los principios, prácticas y valores de la misma.

Los emprendimientos participantes del CEES (así como aquellos que participarán) son potencialmente sustentables ya que si bien presentan debilidades y dificultades en aspectos de calidad y presentación de la producción, estrategias de venta y de difusión para trabajar en otra escala e incorporar nuevas actividades, estas cuestiones se vienen trabajando con las acciones enunciadas anteriormente (talleres de capacitación, cursos a pedido de los emprendedores, espacios de comercialización, y el Banco Solidario). Sin embargo, cabe destacar que es necesaria una mayor amplitud de este proceso para poder incorporar, no solo más emprendedores, sino también profesionales de distintas disciplinas (trabajadores sociales, ingenieros, sociólogos, diseñadores industriales, entre otros) que colaboren en potenciar sinérgicamente a los emprendimientos.Además, los actores públicos involucrados deben trabajar en una propuesta de mediano plazo, pensando en la cimentación de un movimiento colectivo de ESyS en la región.

Según Coraggio:

Un proceso de estas características es imposible que avance a partir del apoyo a emprendimientos aislados (que es una condición necesaria pero no suficiente). Es necesario además hacerlo con una orientación estratégica en función de lo señalado anteriormente, y a la vez impulsar un compromiso de actuación de otras entidades, organizaciones y sectores que aporten en el nivel de las macro relaciones y de las instituciones vinculadas.

Esto implica un punto de partida inicial claro e importante: decisión y apoyo político, además de una fuerte inyección de recursos. En ese marco es clave la participación de la universidad, no sólo en relación con los emprendimientos involucrados, sino también a su vinculación con el sector público y otras organizaciones de la sociedad. (2011:226-227)

Asimismo, se están generando grupos de trabajo entre emprendedores más estables y que se vienen sosteniendo desde hace unos meses. Sin embargo, los mismos necesitan fortalecerse en cuanto a organización interna y profundizar la relación entre ellos. En este punto es fundamental el apoyo y seguimiento que puedan realizar desde las entidades 
públicas vinculadas al CEES, para orientarlos y contenerlos en el complejo camino de la asociatividad y la horizontalidad.

Actualmente las instituciones participantes en el CEES tienen sus dificultades en cuanto al rol que se les pretende otorgar. No hay un acuerdo claro entre los organismos participantes sobre un horizonte de corto y de mediano plazo, falta una continuidad en el planeamiento, prevaleciendo muchas veces el cortoplacismo en la ejecución y gestión diaria de las diversas actividades llevadas a cabo. Una dificultad central que posee el CEES es el tema presupuestario que impide planificar algo bajo este sistema regresivo, altamente dependiente de los gobiernos superiores; dependentismo financiero y paupérrimos circuitos administrativos y fiscales hacen de la necesaria autonomía como prerrequisito para el afianzamiento de las expresiones de la ESyS una realidad muy lejana.

Entre algunos de los inconvenientes que se observan se destacan los siguientes: el clientelismo político, ausencia de generación de información útil para toma de decisiones, falta de motivación de parte del personal afectado al área. Todos son factores que impactan negativamente en el fortalecimiento y expansión del movimiento de la ESyS tanto en el corto como en el mediano plazo.

\section{Propuestas y conclusiones}

El objetivo de este trabajo fue analizar y profundizar el itinerario y las dinámicas desarrolladas en los últimos 3 años en el CEES, indagando las interacciones institucionales y sociales que se han venido generando en este tiempo.

Luego del recorrido del CEES esbozado brevemente en este artículo, se puede mencionar como una reflexión sumamente importante que las experiencias y expresiones de Economía Social y Solidaria de la ciudad necesitan, por un lado, de un conjunto de condiciones favorables a fin de ser sostenibles en el tiempo y, por otro, de un consentimiento y apoyo de
Ios actores sociales, institucionales y civiles implicados en el lugar, con un rol estatal preponderante, a fin de generar un ambiente favorable para el fortalecimiento de la Economía Social. Por sobre todas las cosas, porque actualmente la vida económica y social se desarrolla en un contexto de economía capitalista de fuerte competencia.

Si el sector público provincial, municipal y universitario de Santa Fe pretenden seguir manteniendo su actitud proactiva en esta área de trabajo, incluyendo a los emprendedores sociales y a los cooperativistas en sus agendas de trabajo, es necesario, que los actores implicados previamente cuenten con una sólida y firme base teórica, con un marco analítico de objetivos e instrumentos disponibles (el conjunto de herramientas de intervención) y con un diagnóstico completo de la situación de la ESyS anterior a la ejecución de medidas de acción para de esta manera poder evaluar en un futuro próximolos impactos y consecuencias de los instrumentos de acción puestos en marcha.

Las acciones y medidas que promuevan el Gobierno Provincial, el Municipal y la Universidad desde el CEES deberían cumplir tres funciones: primera, establecer el marco institucional y normativo en el cual van a operar y trabajarlos emprendedores y los cooperativistas; segunda, intervenir activamente en el proceso de fortalecimiento y organización del movimiento de la ESyS de la ciudad; y tercero, lograr el máximo de bienestar económico y social de los emprendedores y cooperativistas.

Cabe recalcar también que si bien hay dificultades y limitaciones para el reconocimiento y el alcance de lo que es la Economía Social, muchos emprendedores y cooperativistas se han comenzado a familiarizar con lo que representa la mismay que encuentran en este campo de experiencias e iniciativas una base a partir de la cual continuar desarrollándose. Como lo expresará uno de los referentes de las cooperativas: AAhora nosotros nos sentimos parte y nos vemos dentro de la Economía Social y vamos a seguir construyendo y defendiendo esto que hemos venido levantando entre todos». 
Por otro lado es necesario generar un conjunto de condiciones en el CEES para que el mismo se comience a convertir en la plataforma de ESyS de la ciudad de Santa Fe y su zona de influencia. Para ello es recomendable lo siguiente:

- En primera instancia, la generación y construcción de un ámbito de trabajo colectivo en el CEES cimentado en una democracia participativa autentica. Para esto se debe asegurar el involucramiento y la participación de todos los actores sociales y públicos involucrados en el CEES, institucionalizando y reglamentando el sistema de participación y toma de decisiones.

- En segunda instancia, es necesario la creación e instrumentación de un marco regulatorio que contemple a los emprendedores sociales y a los cooperativistas que se nucleen en el CEES. Sucede muchas veces que estas iniciativas se orientan a la economía informal porque no existe un ordenamiento legal que las considere. La mayor parte de la estructura legal, jurídica e impositiva que contempla a las prácticas de la ESyS están basadas en la ley de sociedades comerciales y las leyes impositivas están diseñadas e instrumentadas sobre la base de un sistema capitalista de mercado cuyos sujetos contemplados son las unidades de producción movilizadas por la máxima ganancia.

Por lo tanto si bien las prácticas de la ESyS son socialmente legítimas, son jurídicamente ilegales. Este sector socioeconómico debe estar regulado desde la perspectiva legal e impositiva en los distintos niveles del Estado (nacional, provincial y municipal). Por este motivo es que es imprescindible el diseño e instrumentación de normas jurídicas específicas (ordenanzas municipales, leyes provinciales y nacionales) a favor de nuevas formas asociativas (ferias, empresas recuperadas, emprendimientos sociales, producción en asentamientos de pueblos originarios, etc.) y leyes impositivas que favorezcan la producción local basada en la ESyS.
Una alternativa legal que viene ganando visibilidad en los últimos añoses la obligatoriedad por parte de Municipios y Gobiernos provinciales de utilización de insumos de producción local, en restaurantes y supermercados (por ej., compra de producción ictícola) y casas comerciales (exhibición de producción artesanal en góndolas y expositores) a fin de favorecer mayores posibilidades de difusión y comercialización, y la exención de IVA, ganancias, Ingreso brutos, etc., por las compras y ventas de las mismas.

- En tercer lugar la educación es fundamental como instrumento estratégico para el desarrollo de capacidades necesarias para la actividad productiva y la formación en valores básicos. Desde este enfoque es primordial el aporte de las universidades, no sólo en términos de aplicar el conocimiento científico a los procesos productivos, sino en la generación de una masa crítica sobre los efectos negativos del sistema capitalista y la necesidad de otras formas de funcionamiento del mercado. En esta situación el CEES se puede convertir es un espacio de referencia para la educación no solo en la dimensión de los saberes técnicos, sino en un aspecto fundamental como lo es la formación, y fundamentalmente la práctica y coherencia de la Economía Social.

En cuanto a los logros del CEES hay que destacar que supero la focalización de las políticas sociales, ya que se ofrecen una serie de servicios (formación, asesoramiento, espacios de comercialización, banco solidario, entre otros) al conjunto de emprendedores sociales y cooperativistas de la ciudad sin ninguna discriminación ni exclusión, y se tienen en cuenta las demandas y necesidades de los mismos, atendiéndolas a través de acciones concretas, personalizando la atención. Sin embargo, todavía no se han podido diseñar e implementar políticas y medidas integrales (que abarquen cuestiones sociales, culturales, políticas, además de la organizacional) que vayan más allá de la dimensión productiva. Un aspecto limitante en este sentido es que el CEES no cuenta con una asignación presupuestaria perma- 
nente, lo que lleva a que se deban priorizar algunas actividades y acciones en desmedro de otras, llevando a que el impacto que pueda tener el CEES en términos temporales y territoriales tenga serias limitaciones. Por este motivo es que el rol activo y la convicción de las instituciones participantes del CEES deben manifestarse en compromisos institucionales mediante la creación de un convenio de participación de los tres niveles del Estado, más allá de los gobiernos de turno y el financiamiento a estas expresiones mediante la incorporación permanente en el Presupuesto.

Con respecto a la hipótesis del documento, considero que la misma se verifica a lo largo del mismo, puesto que para que se desarrolle y se fortalezca la ESyS es necesaria la decisión y convicción del Estado en sus distintos niveles, además de una fuerte inyección de recursos financieros, técnicos, profesionales, sumado a un constante seguimiento, monitoreo y apoyo por parte de los erarios públicos a las expresiones y experiencias de la ESyS. Queda esbozado el interrogante acerca de las acciones y medidas que permitirían organizar colectivamente estas prácticas de producción alternativas que promuevan nuevas formas de inclusión social para sus miembros, y las funciones, alcances, responsabilidades y organizaciones que se deben dar el Estado y los distintos actores sociales y académicos para acompañar este proceso. 


\section{Referencias bibliográficas}

- Arroyo, D. (2013). La desmunicipalización del Desarrollo Local: perfil productivo, etapas y situación actual en la Argentina. Facultad Latinoamericana de Ciencias Sociales (FLACSO). Curso de Posgrado de Desarrollo Local y Economía Social y Solidaria. Buenos Aires. FLACSO.

- Coraggio, J.L (2004). La gente o el capital. Desarrollo Local y Economía del Trabajo. Buenos Aires: Editorial Espacio.

- - (2005). ¿Es posible otra economía sin (otra) política? El Pequeño Libro Socialista. Buenos Aires: La Vanguardia.

- - (2007). Economía social, acción pública y política (Hay vida después del neoliberalismo). Buenos Aires: CICCUS.

- (2010). Desarrollo regional, espacio local y economía social. V Coloquio Local, III Regional y el II Foro de Economía Social «Economía Social/Desarrollo Local: una tensión a develar en la construcción del buen vivir». Universidad Nacional del Litoral, Santa Fe, Argentina.

- - (2011). Economía social y solidaria. El trabajo antes que el capital. Quito, Ecuador:Abya-Yala.
- García Delgado, D. (2013). Desarrollo y modelo societal. Modelos de acumulación. Crisis global e impacto para Argentina y la Región. Los desafíos para un nuevo modelo. Facultad Latinoamericana de Ciencias Sociales (FLACSO). Curso de Posgrado de Desarrollo Local y Economía Social y Solidaria. Buenos Aires: Flacso.

- Gobierno de la Provincia de Santa $\mathrm{Fe}$ (14 de mayo de 2013). Provincia, Municipalidad y UNL articulan acciones. Recuperado de www.gobierno. santafe.gov.ar/prensa/ImprNoticia pdf.php?idnoticia $=184723$

- Instituto Nacional de Estadísticas y Censos (INDEC). (2016). Encuesta Permanente de Hogares. Recuperado de http://www.indec.gov.ar/basesde-datos.asp

- Hintze, S. (2007). Políticas sociales argentinas en el cambio de siglo. Conjeturas sobre lo posible. Buenos Aires: Espacio.

- Massera, M. (2015). Economía Social y políticas públicas en la ciudad de Santa Fe: el rol del Centro de Emprendedores de la Economía Social. Ponencia presentada en el $12^{\circ}$ Congreso Nacional de Estudios del Trabajo, Buenos Aires, Argentina.
- Mejías, D. (2014). De la autogestión al asociativismo: Elaboración de un Sistema de Apoyo para el acompañamiento a pequeñas unidades productivas en el marco de la Economía Social y Solidaria. Formulario Proyecto de Extensión de Interés Social (PEIS), Convocatoria 2014, Universidad Nacional del Litoral (no publicado).

- Nosetto, L. (2013). Teorías de la Economía Social y el caso de las Cooperativas de Servicios Públicos. Facultad Latinoamericana de Ciencias Sociales (FLACSO). Curso de Posgrado de Desarrollo Local y Economía Social y Solidaria. Buenos Aires: Flacso.

- Polanyi, K. (1989). La Gran Transformación: critica del liberalismo económico. Madrid: Ediciones de La Piqueta.

- Tealdo, J. (2013). Programa de Extensión de Economía Social y Solidaria de la Universidad Nacional del Litoral (no publicado). Universidad Nacional del Litoral. Presentación institucional del Centro de Emprendedores de la Economía Social. Recuperado en Mayo 2016 de http:// www.unl.edu.ar/categories/view/ centro_de_emprendedores1\#. V2w2KfI97IU

\section{Registro bibliográfico} Sotto, 0.M. (2017). El Centro de Emprendedores de la Economía Social (CEES) de la ciudad de Santa Fe y su viabilidad de convertirse en un centro de referencia para la Economía Social de la región santafesina. Revista Ciencias Económicas, 14(01), 167-184. 